\title{
Preparation, Characterization and Measurement of CMC of SDS, CTAB and Tween 80 Based Self-Assembled Aggregates in W/O and O/W Microemulsions as Systems for Preparing Nanomaterials
}

\author{
Rexona Khanom*, Abu Bin Hasan Susan, Md Yousuf Ali Mollah, \\ Abu Noim Munshi Shahidur Rahman
}

Department of Chemistry, University of Dhaka, Dhaka, Bangladesh

Email address:

rexonakhanom@yahoo.com (R. Khanom)

\section{To cite this article:}

Rexona Khanom, Abu Bin Hasan Susan, Md Yousuf Ali Mollah, Abu Noim Munshi Shahidur Rahman. Preparation, Characterization and Measurement of CMC of SDS, CTAB and Tween 80 Based Self-Assembled Aggregates in W/O and O/W Microemulsions as Systems for Preparing Nanomaterials. American Journal of Applied Chemistry. Vol. 3, No. 3, 2015, pp. 105-123. doi: 10.11648/j.ajac.20150303.14

\begin{abstract}
Series of water in oil (w/o) composition of microemulsions were prepared from systems with sodium dodecyl sulphate (SDS), 1-butanol, cyclohexane, water; tween 80, 1-butanol, cyclohexane, water and cetyltrimethylammonium bromide (CTAB), 1-butanol, cyclohexane, water; at varying water to surfactant mole ratio, w0 and oil in water (o/w) composition of SDS and $\mathrm{CTAB}$ based microemulsions were made at reconcile with composition of w/o microemulsions. Micelles and reverse micelles are dominant form of self-assembled aggregates in o/w microemulsions and w/o microemulsions respectively. The ability of these aggregates to solubilize water had been employed for the detection of the critical micellization concentration, $\mathrm{cmc}$ for micelles and reverse cmc for reverse micelles. The reverse cmc values are smaller $(0.204-0.293 \mathrm{M})$ than the micellar $\mathrm{cmc}$ $(0.4605-0.607 \mathrm{M})$ was observed.1-butanol as a stabilizer provided information of the systems to give thermodynamically stable transparent w/o and o/w microemulsion due to its low linear thickness. Specific conductance, density and aggregation number had been used to characterize SDS and CTAB based microemulsions and the microemulsions prepared by non-ionic surfactant, tween 80 were characterized by only density measurement. Lower conductance and density are characteristics for w/o microemulsions having high 1-butanol content while in o/w microemulsions leads higher conductance and density. Steady-State Fluorescence Quenching method had been employed to determine the aggregation number, $\mathrm{N}$ of the organized self-assembled aggregates. The aggregation number of the aggregates in the microemulsions varied with composition of the microemulsions and depends only on the concentration of surfactant present in the systems. Aggregation number had been found to increase as the number of micellar aggregates increases. However, an increase in the number of reverse micellar aggregates in the system brought about gradual decrease of the aggregation number. As the quenching process of SDS based aggregates was very effective, however that of CTAB was less pronounced, the aggregation number of SDS based aggregates had been found to be comparatively lower than that of $\mathrm{CTAB}$ under similar experimental conditions. The results obtained show that $\mathrm{N}$ values which range 78-101 for SDS based aggregates and range 99-160 for CTAB based aggregates show a linear relationship with the concentration of surfactant. Detailed analysis of the conductivity, density and aggregation number, it can be tuned by changing composition of microemulsions and suitable compositions of microemulsions must be employed for the preparation of nanomaterials.
\end{abstract}

Keywords: Preparation, Characterization, SDS, CTAB, W/O and O/W Microemulsions, CMC, Water Solubilization

\section{Introduction}

The preparation and characterization of micro-emulsions have received an upsurge of interest from many researchers in diversified areas for example: Chemistry, Physics,
Nano-material Science, Biology and the corresponding Engineering. Since the microemulsions usually exhibit unusual optical properties, significantly different from those of the emulsions due to their extremely small sizes of self-assembled aggregates and large surface areas, 
thermodynamic stability, ease of preparation, higher solubility and good appearance [1]. During the past few years, preparation of the nanomaterials employing microemulsion systems is emerging as a potential area of interest. Extensive reviews on the physicochemical and electrochemical characterization of microemulsion are available [2-6].

Research to date includes numerous attempts to prepare microemulsions and characterize them. However, there are still challenges such as the instability of the microemulsions, control of their aggregates size and uniform dispersity. It should be noted that w/o microemulsions are transparent, isotropic liquid media with nanosized water droplets that are dispersed in continuous oil phase and stabilized by co-surfactant molecules at the water/oil interface. The $\mathrm{o} / \mathrm{w}$ microemulsions are, on the other hand, transparent, isotropic liquid media with nanosized oil droplets that are dispersed in a continuous aqueous phase and stabilized by cosurfactant molecules (less than w/o microemulsion). The w/o microemulsion systems are dominated by self-assembled reverse micelles and in the $\mathrm{o} / \mathrm{w}$ micro-emulsion systems, self-assembled micelles dominates reverse micelles. Micelles and reverse micelles are dynamic nanoscopic aggregates of surfactant molecules having specific capability to solubilize a variety of molecules (e.g. water) with different hydrophobicities [7-9]. Due to the association in w/o microemulsion, micelles form thermodynamically stable reverse micelles with cosurfactants $[10,11]$ which find a number of interesting applications $[12,13]$. Although micelles and reverse micelles dominated microemulsions can solubilize small amounts of organic compounds in aqueous micellar solutions, [14] the solubilization of water in these solutions has received scant attention in the literature [15]. Practical applications of w/o and o/w microemulsion require more detailed characterization rather than phase transition. The influence of individual aggregate of the microemulsion phases on the overall composition is also important. Only very few detailed investigations on the characterization of w/o and $\mathrm{o} / \mathrm{w}$ microemulsion are available. These include determination of aggregation number of w/o microemulsion by fluorescence measurement [16] and optimization of reverse $\mathrm{cmc}$ of w/o microemulsion with polymeric composite [17]. The ability of the reverse micelles to solubilize water (once they are formed) had been applied for the detection of the critical micellization concentration, cmc. The cmc of amphiphilic molecules in organic solvents cannot be determined from, for example, surface tension measurements [18], since there is no significant tendency of the amphiphiles to adsorb oil at the air-oil interface. Instead, water solubilization can prove useful in the determination of $\mathrm{cmc}$ in organic solvents [19]. A notable increase in the water solubilization capacity (expressed in water molecules per surfactant) was observed when the surfactant content significantly decreased in w/o microemulsion. However, this measurement on the $\mathrm{o} / \mathrm{w}$ microemulsion has no ever been reported in the literature [20].The size, shape, polidispersity, interfacial polarity, microviscosity, aggregation number etc., of reverse micelles are of general importance and a variety of techniques and methods which permits easy and reliable measurements of such parameters are available [21, 22]. Among them, fluorescence quenching has proved to be a powerful tool to obtain information on the compositional, structural dynamic properties of organized assemblies. Important parameters such as aggregation numbers and distribution of probes and quenchers have been evaluated by using either Steady-State Fluorescence Quenching or Time-Resolved Fluorescence Quenching methods (SF and TF respectively) [23]. Both methods use a fluorescent probe and a quencher but meanwhile SF involves measurements of the fluorescence emission intensity at increasing quencher concentration and $\mathrm{TF}$ involves fluorescence decay experiments using commercially available photon counting apparatus.

The literature survey presented above suggests that many interesting questions can still be raised regarding characterization and the correlations of the aggregation number with surfactant concentration and composition of w/o and $\mathrm{o} / \mathrm{w}$ microemulsion. Under fixed weight percentage ratio of surfactant to cyclohexane, how does density, specific conductance and aggregation number of individual microemulsion influence the overall composition of the w/o and $\mathrm{o} / \mathrm{w}$ microemulsion, for example can 1- butanol as cosurfactants influence the density of the microemulsion? How does the density influence the composition of the microemulsion? Can 1-butanol influence the specific conductance of microemulsion? Which component of the microemulsion significantly determines its overall conductivity? How does the specific conductance influence the composition of microemulsion? How does the surfactant concentration affect the cmc values of $\mathrm{w} / \mathrm{o}$ and $\mathrm{o} / \mathrm{w}$ microemulsion? Does the aggregation number depend on the surfactant concentration? What is the effect of concentration of surfactant across a composition of a microemulsion on the aggregation number? How does variation of aggregation number with surfactant concentration across a composition of microemulsion? These are some of the issues are addressed here.

In the present work, series of water in oil (w/o) composition of microemulsions were prepared from systems with sodium dodecyl sulphate (SDS), 1-butanol, cyclohexane, water; tween 80, 1-butanol, cyclohexane, water and cetyltrimethylammonium bromide (CTAB), 1-butanol, cyclohexane, water; at varying water to surfactant mole ratio, w0 and oil in water $(\mathrm{o} / \mathrm{w})$ of SDS and CTAB based composition of microemulsions were made at reconcile with composition of w/o microemulsions. The surfactant/cyclohexane ratio of w/o microemulsions was kept at a constant. The microemulsions thus prepared were characterized by measuring the density, conductivity and aggregation number. Some interesting observations and generalizations are summarized here. We had employed Steady-State Fluorescence method to measure micellar concentration in solution, [mic] and reverse micellar concentration in solution, [reverse mic] of micelle dominated $\mathrm{o} / \mathrm{w}$ microemulsion and reverse micelle dominated w/o microemulsion respectively formed by systems with sodium 
dodecyl sulphate (SDS),1-butanol, cyclohexane, H2O and cetyltrimethylammonium bromide (CTAB), 1-butanol, cyclohexane, H2O. The effect of 1-butanol content on specific conductance of SDS and CTAB based microemulsions; the effect of 1-butanol content on densities of SDS, CTAB and tween 80 based microemulsions; the effect of composition of microemulsions on the specific conductance and the density; the effect of surfactant concentration on the aggregation number of micelles and reverse micelles in the $\mathrm{o} / \mathrm{w}$ and $\mathrm{w} / \mathrm{o}$ microemulsion systems; the effect of surfactant concentration across composition of microemulsion on the aggregation number and to gain insight into the characteristic variation of the aggregation number within a composition of SDS and CTAB based microemulsions are investigated here.

\section{Materials and Methods}

\subsection{Materials}

Cetyltrimethylammonium bromide (CTAB), sodium dodecyl sulphate (SDS) and tween 80 were kindly supplied by E. Merck. 1-butanol and cyclohexane were used as received without further purifications. N-Cetylpyridiniumchloride was used as quencher purchased from Sigma Aldrich and pyrene was also from Sigma Aldrich, used as the fluorescent probe. Deionized water was used in all preparations.

\subsubsection{Preparation of Stock Solution}

Stock solution of $1 \mathrm{M}$ quencher was prepared by dissolving a required amount of N-Cetylpyridiniumchloride in volumetric flask and made up to the mark using de-ionized water. Stock solution of $2 \times 10-4 \mathrm{M}$ probes was prepared by dissolving a required amount of pyrene in volumetric flask and made up to the mark using absolute ethanol. The solutions for the fluorescence measurement were prepared with special care. From a stock solution of $2 \times 10-4 \mathrm{M}$ pyrene in absolute ethanol, a known volume $(50 \mu \mathrm{L})$ was pipetted into a reagent bottle and kept it two nights for evaporating the ethanol. The final concentration of pyrene in the solution was thus $2 \mu \mathrm{M}$. After evaporating the ethanol, $10 \mu \mathrm{L}$ quencher solution was pipetted into pyrene containing reagent bottle and then $5 \mathrm{~mL}$ microemulsions added into the reagent bottle. By mixing the surfactant and pyrene solutions without quencher and with quencher in 5 or 6 solutions varying in quencher concentration from 0 to maximum concentration were prepared so that the concentration of total surfactant concentration present in microemulsions was not change.

\subsubsection{Preparation of $\mathrm{W} / \mathrm{O}$ and $\mathrm{O} / \mathrm{W}$ Microemulsion}

SDS based w/o microemulsions were prepared at a fixed CTAB to cyclohexane ratio with different proportion of water, 1-butanol, water to surfactant ratio, wo ranging from 15 to 33; CTAB and tween 80 based microemulsions were also prepared with w0 ranging from 15 to 35 are presented in Table 1, Table 2 and Table 3 respectively. And SDS and CTAB based $\mathrm{o} / \mathrm{w}$ microemulsions were prepared with different proportion of water, 1-butanol and cyclohexane reconcile with composition of w/o microemulsions are inserted in Table 4,
Table 5 respectively.

\subsection{Methods}

\subsubsection{Density, Conductance and Fluorimetric Measurement of Microemulsion}

Macroscopic density values for both micelle and reverse micelle dominated solution were measured by employing weighing balance and reported values inserted in Table 6 are an average of 12 measurements. A mixture of known weights of surfactant, co-surfactant and oil was placed in the beaker at a constant temperature. The conductivity of ionic SDS and CTAB based microemulsions were measured to an accuracy of $0.5 \%$ using a digital conductivity meter (J.P Selecta, Abrera; Spain, Model no CD-2005) is shown in Table 7. The Fluorimetric measurement setup was carried out using a Fluorescence Spectrophotometer (Hitachi; Model no F-7000); the sensitivity of the equipment was 1 ; rectangular quartz cells were used throughout the experiment to irradiate ultraviolet light for the excitation of pyrene. The excitation and emission slit width was maintained at $5.0 \mathrm{~nm}$. The emission spectrum was scanned over the range $340-480 \mathrm{~nm}$ for reverse micelles and that of $360-480 \mathrm{~nm}$ for micelles. The excitation wavelength was $360 \mathrm{~nm}$ and the pyrene monomer emission was measured at $395 \mathrm{~nm}$ of reverse micelle dominated w/o microemulsions and at $375 \mathrm{~nm}$ of micelle dominated $\mathrm{o} / \mathrm{W}$ microemulsions. In Steady-State Fluorescence measurements, the investigated micellar and reverse micellar systems were continuously irradiated and the spectrum of the emitted light (fluorescence) were recorded. To extract information about physical property e.g. aggregation number of the systems, we followed the method proposed by Turro and Yekta [24, 25].To storage data from fluorescence quenching, the Fluorescence Spectrophotometer used an attached computer. The logarithm of the recorded intensity ratio I0/IQ was calculated at different quencher concentration. We had chosen the intensity of third peak of the vibronic bands of pyrene to minimize scattering problems. Steady-state fluorescence quenching in homogeneous solutions is described by the well-known Stern-Volmer relationship of equation here [26, 27].

$$
\mathrm{F}=\mathrm{T} 0 \mathrm{~F} 0 / \mathrm{T}=1+\mathrm{kq} \text { T0 [Q] }
$$

where, F0, F, T0 and T are the unquenched and quenched fluorescence intensities and life-times respectively. It should be pointed out that the above equation is valid only for the case of dynamic collisional quenching, whereas if the quenching is due to ground-state complex formation then $\mathrm{T} 0=$ $\mathrm{T}$. Also recall that $\mathrm{kq}$ in isotropic media is a second-order rate constant expressed in M-1 s-1. From the above equation, it becomes evident that long $\mathrm{T} 0$ favors the quenching experiment by improving the experimental accuracy. In micellar media, steady-state fluorescence quenching has been studied by several Investigators. When both the fluorophore and the quencher are immobile, the situation can be described by equation is shown below.

$$
\mathrm{F} 0 / \mathrm{F}=\{[\mathrm{Q}] /[\mathrm{M}]-1 /(\mathrm{R}+\mathrm{i}) \mathrm{i} !\} \exp ([\mathrm{Q}] /[\mathrm{M}]) / \mathrm{R}
$$


where, $\mathrm{R}$ is the ratio of the rate constant of the unquenched deactivation $\mathrm{k} 0$, divided by the quenching rate constant $\mathrm{kq},[\mathrm{M}]$ is the micellar concentration in solution, defined by the following equation and $\mathrm{i}$ express the number of quenchers residing in a micelle [28].

$$
[\mathrm{M}]=([\mathrm{Cs}]-[\mathrm{CMC}]) / \mathrm{Ns}
$$

It is obvious that in order to obtain any information from the fluorescence quenching experiment it is necessary that the fluorescence quenching rate constant $\mathrm{kq}$, is larger, or at least comparable to the fluorescence rate constant $\mathrm{k} 0$. If this is not the case then the system will fluoresce before the quencher has enough time to modify the characteristics of the emission, and therefore very little information will be extracted from fluorescence quenching. When $\mathrm{R}=0$, i.e. in the ideal case of a fluorophore with very long fluorescence life-time ( $\mathrm{k} 0$ is very small) in conjunction with a very efficient quencher ( $\mathrm{k} 0$ is very large), the above 2nd equation can be written approximated by the equation is shown below.

$$
\ln (\mathrm{F} 0 / \mathrm{F})=[\mathrm{Q}] /[\mathrm{M}]
$$

The slope of the line obtained by plotting $\ln (\mathrm{F} 0 / \mathrm{F})$ versus [Q] according to equation (2) is equal to $1 /$ [M]. Therefore when $\mathrm{R}=\mathrm{O}$ the micellar concentration in solution, $[\mathrm{M}]$ can be obtained from steady-state fluorescence quenching experiment, in practice, when $\mathrm{R}$ is small, equation (2) can be applied with confidence as far as the reliability of the Ns numbers obtained is concerned.

\subsubsection{Water Solubilization}

Microemulsions were prepared individually in the 3-25 wt. \% (for w0 $=15$ of SDS based) and $8-13.5 \mathrm{wt} \%$ (for $\mathrm{o} / \mathrm{w}$ microemulsion-1 of SDS based) concentration range by weighing. The microemulsions were carefully sealed with caps to avoid solvent evaporation. Water was titrated to the microemulsions in increments of $\sim 0.0353 \mathrm{~g}$. Following the addition of each water drop, the samples were homogenized by mixing and left standing for $15-30 \mathrm{~min}$ in order to equilibrate which was illustrated in Table 8 and Table 9 in case of SDS based microemulsions. If the resulting solutions were homogeneous and transparent, more water was added. The development in the samples of a hazy or milky appearance after the addition of a water drop was an indication that the maximum solubilization was reached resulting in a phase separation of the samples to an aqueous and oil phase. A majority oil-rich phase separation into w/o microemulsion and a minority aqueous-rich phase into o/w microemulsion were occurred. Microemulsions were occasionally added to these hazy and milky samples in order to clear them up (by decreasing the water concentration) and ensure the reversibility of the phase separation. Similar process for CTAB based $\mathrm{w} / \mathrm{o}$ and $\mathrm{o} / \mathrm{w}$ microemulsions but varying compositions is shown in Table 10 and Table 11.

Table 1. Different composition of SDS based w/o microemulsion.

\begin{tabular}{llllll}
\hline \multirow{2}{*}{ w/o microemulsion } & \% wt. of & & & cyclohexane & water \\
\cline { 2 - 6 } & SDS & 1-butanol & 47.1 & 14 & 18.9 \\
w0 \\
2 & 20 & 40.8 & 14 & 25.2 & 15 \\
3 & 20 & 37.02 & 14 & 29.98 & 20 \\
4 & 20 & 28.2 & 14 & 37.8 & 30 \\
5 & 20 & 24.42 & 14 & 41.58 & 33 \\
\hline
\end{tabular}

Table 2. Different composition of CTAB based w/o microemulsion.

\begin{tabular}{llllll}
\hline \multirow{2}{*}{ w/o microemulsion } & \% wt. of & & & & \\
\cline { 2 - 6 } & CTAB & 1-butanol & cyclohexane & water & \\
\hline 1 & 20 & 50.5 & 16 & 13.5 & 15 \\
2 & 20 & 46.0 & 16 & 18.0 & 20 \\
3 & 20 & 43.3 & 16 & 20.7 & 23 \\
4 & 20 & 37.0 & 16 & 27.0 & 30 \\
5 & 20 & 32.5 & 16 & 31.5 & 35 \\
\hline
\end{tabular}

Table 3. Different composition of tween 80 based w/o microemulsion.

\begin{tabular}{llllll}
\hline \multirow{2}{*}{ w/o microemulsion } & \% wt. of & & & & \\
\cline { 2 - 5 } & tween 80 & 1-butanol & cyclohexane & water & \\
\hline 1 & 20 & 63.95 & 1.2 & 4.05 & 15 \\
2 & 20 & 62.6 & 1.2 & 5.4 & 20 \\
3 & 20 & 61.79 & 1.2 & 6.21 & 23 \\
4 & 20 & 59.9 & 1.2 & 8.1 & 30 \\
5 & 20 & 58.55 & 1.2 & 9.45 & 35 \\
\hline
\end{tabular}


Table 4. Different composition of SDS based o/w microemulsion.

\begin{tabular}{|c|c|c|c|c|}
\hline \multirow{2}{*}{ Microemulsion } & \multicolumn{4}{|c|}{$\%$ wt. of } \\
\hline & SDS & 1-butanol & cyclohexane & water \\
\hline 1 & 20 & 9 & 10 & 61 \\
\hline 2 & 20 & 7 & 8 & 65 \\
\hline 3 & 20 & 5 & 6 & 69 \\
\hline 4 & 20 & 3 & 4 & 73 \\
\hline 5 & 20 & 1 & 2 & 77 \\
\hline
\end{tabular}

Table 5. Different composition of CTAB based o/w microemulsion.

\begin{tabular}{lllll}
\hline \multirow{2}{*}{ microemulsion } & \% wt. of & & \\
\cline { 2 - 5 } & CTAB & 1-butanol & cyclohexane & water \\
\hline 1 & 20 & 14 & 15 & 51 \\
2 & 20 & 12 & 13 & 55 \\
3 & 20 & 10 & 11 & 63 \\
4 & 20 & 8 & 9 & 67 \\
5 & 20 & 6 & 7 & 71 \\
\hline
\end{tabular}

Table 6. Density measurement of SDS based microemulsion.

\begin{tabular}{lll}
\hline SDS based microemulsion & & Density, g/L \\
\hline & 15.0 & 917.6 \\
w/o & 20.0 & 923.8 \\
& 23.0 & 994.0 \\
& 30.0 & 1223.0 \\
& 33.0 & 1229 \\
& 1 & 1087.2 \\
o/w & 2 & 1187.5 \\
& 3 & 1335.5 \\
& 4 & 1367.0 \\
\hline
\end{tabular}

Table 7. The specific conductance of SDS based microemulsions.

\begin{tabular}{lll}
\hline SDS based microemulsion & & Specific conductance (mScm-1) \\
\hline & 15 & 1.52 \\
w/o & 20 & 3.0 \\
& 23 & 4.5 \\
& 30 & 7.95 \\
& 33 & 10.4 \\
o/w & 1 & 13.1 \\
& 2 & 16.4 \\
& 3 & 19.3 \\
\hline
\end{tabular}

Table 8. Water solubilization of SDS based w/o microemulsions with $w 0=15$.

\begin{tabular}{|c|c|c|c|c|c|c|c|}
\hline \multirow{2}{*}{ microemulsions } & \multicolumn{4}{|c|}{$\%$ wt. of } & \multirow{2}{*}{$\begin{array}{l}\text { The amount of } \\
\text { water solubilized, } g\end{array}$} & \multirow{2}{*}{$\begin{array}{l}\text { The amount of water solubilized } \\
\text { per gram SDS }\end{array}$} & \multirow{2}{*}{$\begin{array}{l}\text { Reverse cme } \\
\text { molL-1 }\end{array}$} \\
\hline & SDS & 1-butanol & cyclohexane & water & & & \\
\hline 1 & 3.0 & 47.1 & 14 & 35.9 & 4.16 & 1.39 & \multirow{9}{*}{0.20425} \\
\hline 2 & 5.0 & 47.1 & 14 & 33.9 & 6.96 & 1.39 & \\
\hline 3 & 7.5 & 47.1 & 14 & 31.4 & 36.88 & 4.92 & \\
\hline 4 & 10.0 & 47.1 & 14 & 28.9 & 79.4 & 7.94 & \\
\hline 5 & 12.5 & 47.1 & 14 & 26.4 & 153.6 & 12.29 & \\
\hline 6 & 15.0 & 47.1 & 14 & 23.9 & 511.6 & 34.11 & \\
\hline 7 & 17.5 & 47.1 & 14 & 21.4 & 728.8 & 41.64 & \\
\hline 8 & 20.0 & 47.1 & 14 & 18.9 & 838.4 & 41.92 & \\
\hline 9 & 25.0 & 47.1 & 14 & 13.9 & 1024.4 & 40.98 & \\
\hline
\end{tabular}


Table 9. Water solubilization of SDS based o/w microemulsions-1.

\begin{tabular}{|c|c|c|c|c|c|c|c|}
\hline \multirow{2}{*}{ Microemulsion } & \multicolumn{4}{|c|}{$\%$ wt. of } & \multirow{2}{*}{$\begin{array}{l}\text { The amount of } \\
\text { water solubilized, } g\end{array}$} & \multirow{2}{*}{$\begin{array}{l}\text { The amount of water solubilized per } \\
\text { gram SDS }\end{array}$} & \multirow{2}{*}{$\begin{array}{l}\text { cme } \\
\mathbf{M}\end{array}$} \\
\hline & SDS & 1-butanol & cyclohexane & water & & & \\
\hline 1 & 8 & 9 & 10 & 73.0 & 40.56 & 5.07 & \\
\hline 2 & 8.5 & 9 & 10 & 72.5 & 43.09 & 5.07 & \\
\hline 3 & 9.0 & 9 & 10 & 71.9 & 45.63 & 5.07 & \\
\hline 4 & 9.5 & 9 & 10 & 71.4 & 48.16 & 5.07 & \\
\hline 5 & 10.0 & 9 & 10 & 70.9 & 50.7 & 5.07 & \\
\hline 6 & 10.2 & 9 & 10 & 70.4 & 53.04 & 5.2 & \\
\hline 7 & 10.5 & 9 & 10 & 69.9 & 57.75 & 5.5 & 0.4605 \\
\hline 8 & 11.0 & 9 & 10 & 69.4 & 75.9 & 6.9 & \\
\hline 9 & 11.5 & 9 & 10 & 68.9 & 87.4 & 7.60 & \\
\hline 10 & 12.0 & 9 & 10 & 68.4 & 95.04 & 7.92 & \\
\hline 11 & 12.5 & 9 & 10 & 67.9 & 102.0 & 8.16 & \\
\hline 12 & 13.0 & 9 & 10 & 67.4 & 106.99 & 8.23 & \\
\hline 13 & 13.5 & 9 & 10 & 66.9 & 115.42 & 8.55 & \\
\hline
\end{tabular}

Table 10. Water solubilization of CTAB based w/o microemulsions with $w 0=15$.

\begin{tabular}{llllllll}
\hline \multirow{2}{*}{ microemulsion } & \multicolumn{2}{l}{ \% wt. of } & & $\begin{array}{l}\text { The amount of } \\
\text { water solubilized, } \mathbf{g}\end{array}$ & $\begin{array}{l}\text { The amount of water solubilized } \\
\text { per gram SDS }\end{array}$ & $\begin{array}{l}\text { Reverse cme } \\
\text { M }\end{array}$ \\
\cline { 2 - 5 } & CTAB & 1-butanol & cyclohexane & water & 11.60 & 2.11 \\
\hline 1 & 5.5 & 50.5 & 16 & 28.0 & 2.60 & 2.11 \\
2 & 6.0 & 50.5 & 16 & 27.5 & 12.66 & 2.11 \\
4 & 6.5 & 50.5 & 16 & 26.0 & 13.71 & 2.11 \\
5 & 7.0 & 50.5 & 16 & 25.5 & 14.77 & 3.87 \\
6 & 7.2 & 50.5 & 16 & 25.3 & 27.86 & 3.23 \\
7 & 7.4 & 50.5 & 16 & 25.1 & 23.90 & 4.54 \\
8 & 8.7 & 50.5 & 16 & 24.9 & 39.50 & 4.90 \\
9 & 9.0 & 50.5 & 16 & 24.6 & 44.10 & 5.45 \\
\hline
\end{tabular}

Table 11. Water solubilization of CTAB based o/w microemulsions-1.

\begin{tabular}{|c|c|c|c|c|c|c|c|}
\hline \multirow{2}{*}{ Microemulsion } & \multicolumn{4}{|c|}{$\%$ wt. of } & \multirow{2}{*}{$\begin{array}{l}\text { The amount of } \\
\text { water solubilized, } g\end{array}$} & \multirow{2}{*}{$\begin{array}{l}\text { The amount of water solubilized } \\
\text { per gram SDS }\end{array}$} & \multirow{2}{*}{$\begin{array}{l}\text { Micellar } \\
\text { cmc, } \mathbf{M}\end{array}$} \\
\hline & СТАВ & 1-butanol & cyclohexane & water & & & \\
\hline 1 & 11.0 & 14 & 15 & 60.0 & 67.1 & 6.10 & \\
\hline 2 & 11.5 & 14 & 15 & 59.5 & 70.15 & 6.10 & \\
\hline 3 & 11.7 & 14 & 15 & 59.3 & 71.37 & 6.10 & \\
\hline 4 & 12.0 & 14 & 15 & 59.0 & 73.2 & 6.10 & \\
\hline 5 & 12.2 & 14 & 15 & 58.8 & 97.36 & 7.98 & 0.1995 \\
\hline 6 & 12.4 & 14 & 15 & 58.6 & 106.27 & 8.57 & \\
\hline 7 & 12.7 & 14 & 15 & 58.3 & 117.35 & 9.24 & \\
\hline 8 & 13.0 & 14 & 15 & 58.0 & 129.09 & 9.93 & \\
\hline 9 & 13.5 & 14 & 15 & 57.5 & 145.93 & 10.81 & \\
\hline
\end{tabular}

\section{Results and Discussion}

\subsection{Density and Conductivity Measurements}

By applying data of Table 6 , the variation of density with \% wt. 1-butanol for fives water to surfactant ratios, w0 of SDS and CTAB based microemulsions is placed in Figure 1 which results the densities of microemulsions increase with decreasing \% wt. of 1-butanol. At higher 1-butanol content (47.1\%), the density of SDS microemulsions is $917.6 \mathrm{~g} / \mathrm{L}$ and the density reached the maximum $(2719.0 \mathrm{~g} / \mathrm{L})$, when the 1-butanol content was $24.42 \%$. The density decreases with 
increasing the 1-butanol content across a composition of SDS and CTAB based microemulsion is also allowed in Figure 2. According to our density results, we can say that the density only depend on the wt $\%$ of 1-butanol not the water to surfactant mole ratio, w0. It is possible to discuss this fact by the layer thickness of oil phase and low density of 1-butanol $(0.8089 \mathrm{~g} / \mathrm{cm} 3)$ than water $(0.993973 \mathrm{~g} / \mathrm{cm} 3)$ at $250 \mathrm{C}[16]$. Across a composition, in w/o region, the micellar aggregates form without the presence of 1-butanol as a co-surfactant resulting thinner thickness of oil phase and thus gives large density values, but with the increasing 1-butanol content, significant unstable micelles form stable reverse micelles by association with 1-butanol as a co-surfactant leading less thin thickness of oil phase. However, in $\mathrm{o} / \mathrm{w}$ region, micellar aggregates form highly with a few reverse micellar aggregates, giving low density value initially (1087.2g/L for SDS based). This means that 1-butanol is not only a dispersant medium but also a cosurfactant in these mentioned systems.

By fitting data from Table 7, the results are displayed in Figure 3 . The increase in conductivity is due to the phenomena known as percolation of charges through the droplet cores. The conductivity of SDS and CTAB based microemulsions increases with decreasing $\%$ wt. of 1-butanol indicating that an increase in the number of conducting species in the microemulsions. The degree of ionization of the reverse micellar headgroups is greatly depended on the presence of 1-butanol. At higher \% wt. of 1-butanol (47.1\%), the core droplet comprised of mainly hydrophilic part of sodium dodecyl sulphate which was less dissociated and stabilized by a significant change in the degree of ionization and hence the conductivity was low $(1.51 \mathrm{mScm}-1)$. When the amount $\%$ wt. of 1-butanol decreased, the droplet becomes less stabilized, then it is easy to exchange charges between the droplets and as a consequence, conductivity increased (10.4 mScm-1). The conductivity decreases with increasing the 1-butanol content across a composition of SDS and CTAB based microemulsion is given in Figure 4. Because, as a similar manner of density results in a composition, the conductivity decreases with increasing $w t \% 1$-butanol content implies that thin layer of oil phase brings about large intermicellar ionic exchange [29].

\subsection{Determination of the [Mic] From Fluorescence Quenching Data}

As the third vibronic peak in the emission spectra of pyrene is a sensor of the microenvironmental polarity of the pyrene molecule, we measured steady-state spectra for SDS and CTAB based reverse micelle dominated w/o microemulsions at varying surfactant concentration. The results are summarized in Table 8 and Table 10 and the spectra in case of $\mathrm{w} 0=15$ are inserted in Figure 5. It is very striking that the spectra for the CPC containing reverse micellar solutions are almost identical with the spectra obtained in reverse micellar solutions only. The results confirm that we do have reverse micelles in the presence of $\mathrm{CPC}$, whereas the change in third peak at addition of CPC are much larger for SDS and CTAB based micelle dominated $\mathrm{o} / \mathrm{w}$ microemulsion than that of $\mathrm{w} / \mathrm{o}$ microemulsion. In this case, the interaction was found to be markedly reduced, due to the high surfactant monomer concentration, which contributes to the screening of the electrostatic interactions. The results are addressed in Table 9 and Table 11 and the shapes of the curves in case of microemulsion-1 are explained in Figure 7. And equation (2) predicts that a plot of $\ln$ (I0 / IQ) versus quencher concentration, [Q] should be linear with a slope $=1 /$ [mic]. Some representative plots for the investigated reversed micelles and micelles are given in Figure 6 and Table 8 respectively from where the [mic] values at different surfactant concentrations were obtained. The linear relationship indicates that only one type of quenchingoccurs over a concentration range used in the experiments.

\subsection{Determination of the Reverse Cmc and Micellar Cmc by Water Solubilization Method}

The ability of the both reverse micelles and micelles to solubilize water had been employed for the detection of the critical micellization concentration, cmc. The magnitude of the water solubilization capability depends on the surfactant concentration. When the micellar aggregates started to form, then the aggregates get the capability of solubilizing water. So the concentration at which solubilization starts is called the critical micellization concentration, cmc. The reverse micellar cmc values are smaller than the micellar $\mathrm{cmc}$, because in the w/o microemulsion, the surfactant content is greater than that of o/w microemulsion, thus dissolves a small amount of water. So, the cmc decreased with increasing surfactant concentrations in a composition. A significant increase in the water solubilization capacity (expressed in water molecules per surfactant) was observed. This increase is a process which describes the self-association of the surfactant molecules into micelles or reverse micelles, which provides a hydrophilic microenvironment suitable for water solubilization. In this concentration region, the surfactant molecules that participate in the micelles or reverse micelles should be in equilibrium with monomers. The reverse $\mathrm{cmc}$ and micellar cmc values of SDS based systems are presented in Table 12 and Table 13 respectively. Similarly for CTAB based w/o and $\mathrm{o} / \mathrm{w}$ microemulsions are shown in Table 14 and Table 15.

\subsubsection{Determination of the Reverse Cmc of $w / o$ Microemulsion at $\mathrm{WO}=15$}

The amount of water solubilized over a wide range of surfactant concentrations of SDS based w/o microemulsion at $\mathrm{w} 0=15$ is given in Figure 9. It was demonstrated that no significant amounts of water can be solubilized $(6.9 \mathrm{~g}$ for $\mathrm{w} 0=15$ and $50.7 \mathrm{~g}$ for microemulsion-1) only when the surfactant backbone was in monomer and concentration was very short. Before the cmc point in case of microemulsion at $\mathrm{w} 0=15$, the surfactant molecules was in the form of monomers and high in concentration thus just at this point the aggregates were started solubilization of water in a small amount (36.88 g) resulting low reverse cmc vale $(0.20425$ molL-1). A sudden increase in the water solubilization capability reveals that at cmc point, aggregate formation started when surfactant concentration was $5.0 \mathrm{wt} \%$. At higher concentration of 
surfactant brings that the cmc increases strongly with the surfactant concentration. In this concentration region, the surfactant molecules that participate in the reverse micelles should be also in equilibrium with monomers. After a stage, a plateau was reached at higher surfactant concentrations where the amount of water solubilized per gram of surfactant did not change further with increasing surfactant concentration. Note that the upper surfactant concentration studied here was limited to $20 \mathrm{wt} \%$ for w/o microemulsion of SDS based at $\mathrm{w} 0=15$. Similarly for CTAB based microemulsion is presented in Figure 10.

\subsubsection{Determination of the Micellar Cmc of Microemulsion-1}

The amount of water solubilized over a wide range of surfactant concentrations of SDS based o/w microemulsion-1 is given in Figure 9. It was also demonstrated that no significant amounts of water can be solubilized (50.7 $\mathrm{g}$ for microemulsion-1) only when the surfactant backbone was in monomer and concentration was very small. It was addressed in microemulsion-1 that at cmc point, the surfactant concentration is relatively small, thus solubilize large amount of water $(53.04 \mathrm{~g})$ leading higher cmc value $(0.4605 \mathrm{molL}-1)$. A sudden increase in the water solubilization capability reveals that at cmc point, aggregate formation started when surfactant concentration was $10.0 \mathrm{wt} \%$ for $\mathrm{o} / \mathrm{w}$ microemulsions. At higher concentration of surfactant brings that the cmc increases strongly with the surfactant concentration. In this concentration region, the surfactant molecules that participate in the micelles should be also in equilibrium with monomers. After a stage, a plateau was also reached at higher surfactant concentrations where the amount of water solubilized per gram of surfactant did not change further with increasing surfactant concentration. Note that the upper surfactant concentration studied here was limited to12.8 wt $\%$ for $\mathrm{o} / \mathrm{w}$ microemulsion of SDS based. Similarly for CTAB based o/w microemulsion-1 is inserted in Figure 10.

\subsection{Determination of the Aggregation Numbers of Micelles and Reverse Micelles}

From Figure 5 and Figure 7, fittings to the data, we obtained the information about aggregation number. From each decay curve, the [mic] for the reverse micelles and micelles was obtained. These values obtained at different quencher concentrations were then used to calculate the aggregation number are presented in Tables 16 and Table 17. Regarding quencher free solutions and with solutions, the both micelles follow the same trend, $\mathrm{N}$ increases slightly with increasing surfactant concentration. For SDS based w/o microemulsion, the aggregation number in a $0.636 \mathrm{M}$ solution was determined to about 30 and that of $\mathrm{o} / \mathrm{W}$ microemulsion in $0.754 \mathrm{M}$ solution was 78. The aggregation number of SDS based reverse micelles and micelles increases with increasing surfactant concentration are placed in Table11. The results obtained show that $\mathrm{N}$ values, which range 30-42 and that for micelles range 78-101, indicating $\mathrm{N}$ increases slightly with increasing surfactant concentration and the presence of pyrene and quencher does not change the size of the aggregates. Figure 11 in which a linear relationship between $\mathrm{N}$ and [SDS] exists and extrapolation of the $\mathrm{N}$ values to $[\mathrm{SDS}]=0$ gives an intercept value $\mathrm{N}=0$ proves that in the absence of surfactant no aggregates can be formed. Again in a similar way, aggregation numbers of CTAB based microemulsion systems had been studied are shown in Figure 12 and the results obtained inTable12 which range 39-55 and 99-160 respectively.

\subsection{Effect of Concentration of Surfactant Across $A$ Composition on the Aggregation Number}

In a w/o composition of microemulsion, after the cmc point, surfactants tend to form micelles with only surfactants following reverse micelles with cosurfactants. As a result in a w/o composition, reverse micelle dominated region comes beyond micelle dominated region is put in Figure 13. Aggregation number increases linearly with increasing concentration of SDS across a composition but at a stage the aggregation number starts decreasing with decreasing surfactant concentration is also described in Figure 14. Because, the composition when SDS concentration (0.754-0.964M), passes through the micellar region where the concentration of SDS increases linearly and there is no cosurfactants present. But, the composition when SDS concentration (0.852-0.636M), decreases gradually runs through the reverse micellar region, thus the aggregation number is decreased gradually, because there is surfactant present with cosurfactants. Similarly for CTAB based composition.

\subsection{The Variation of Aggregation Numbers to the Composition of Microemulsion}

The variation of aggregation number with compositions of SDS and CTAB based microemulsions is inserted in Figure 15 which demonstrates that the aggregation number increases as the number of micellar aggregates increases in a composition and then however, an increase of the number of reverse micellar aggregates in the system brings about gradual decrease of the aggregation number. From estimated result, the aggregation number increases in a micellar region and then decreases in a reverse micellar region. Due to the reasons of the rather low linear thickness of 1-butanol, brings relatively smaller size of reverse micelles than that of micelles. Taking into account this concept, the aggregation number of micelles is larger than that of reverse micelles in a microemulsion by the combination of micelle dominated $\mathrm{o} / \mathrm{w}$ composition and reverse micelle dominated w/o composition of each surfactant, SDS and CTAB based. We demonstrate that the aggregation numbers only depend on the surfactant concentration not the concentration of co-surfactant [30]. And from the fluorescence result, we get that the quenching process of SDS based aggregates is very effective, however that of CTAB based aggregates is less pronounced, thus the aggregation number of SDS based aggregates has been found to be comparatively lower than that of CTAB under similar experimental conditions. 


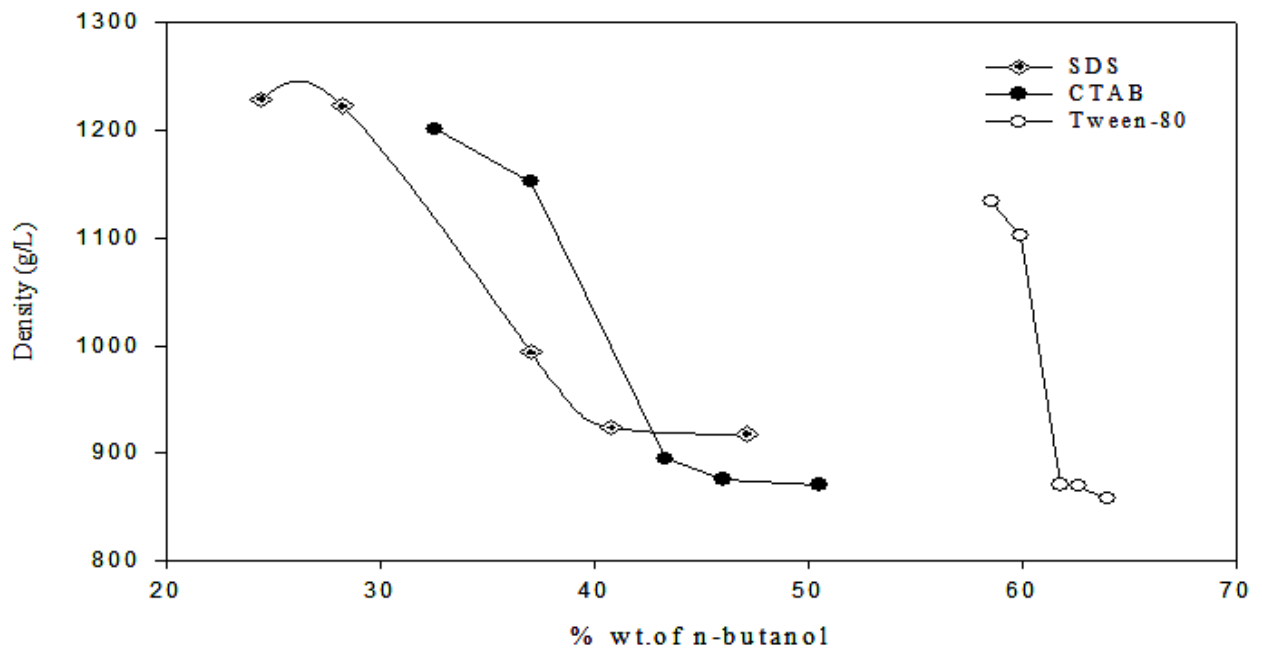

Figure 1. Effect of 1-butanol on the density at varying water to surfactant mole ratio, w0.

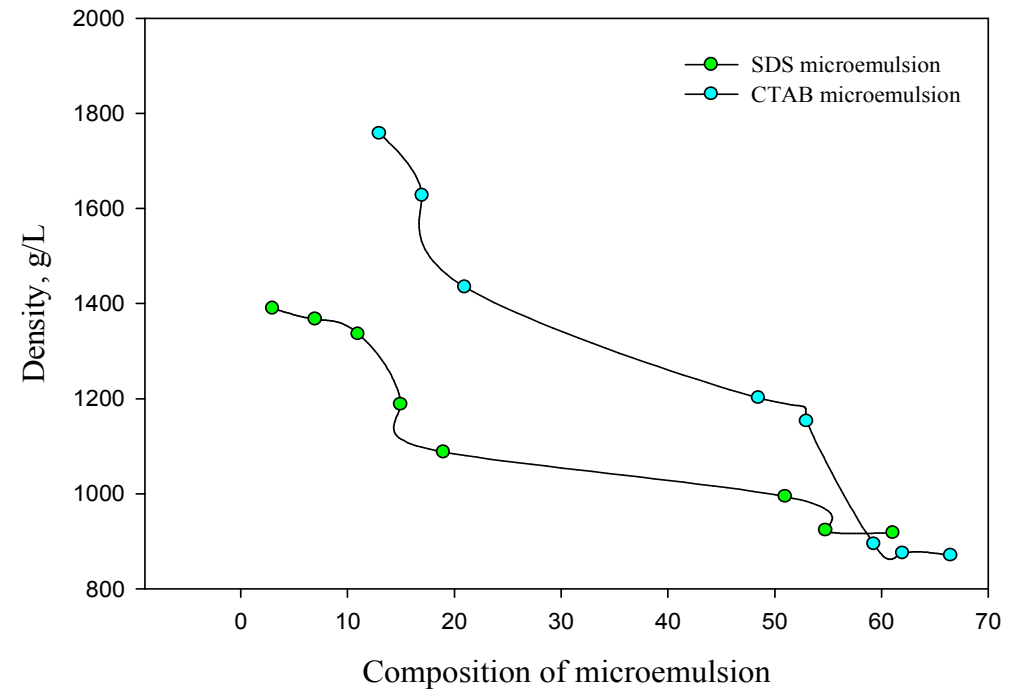

Figure 2. Variation of density with composition of SDS and CTAB based microemulsion.

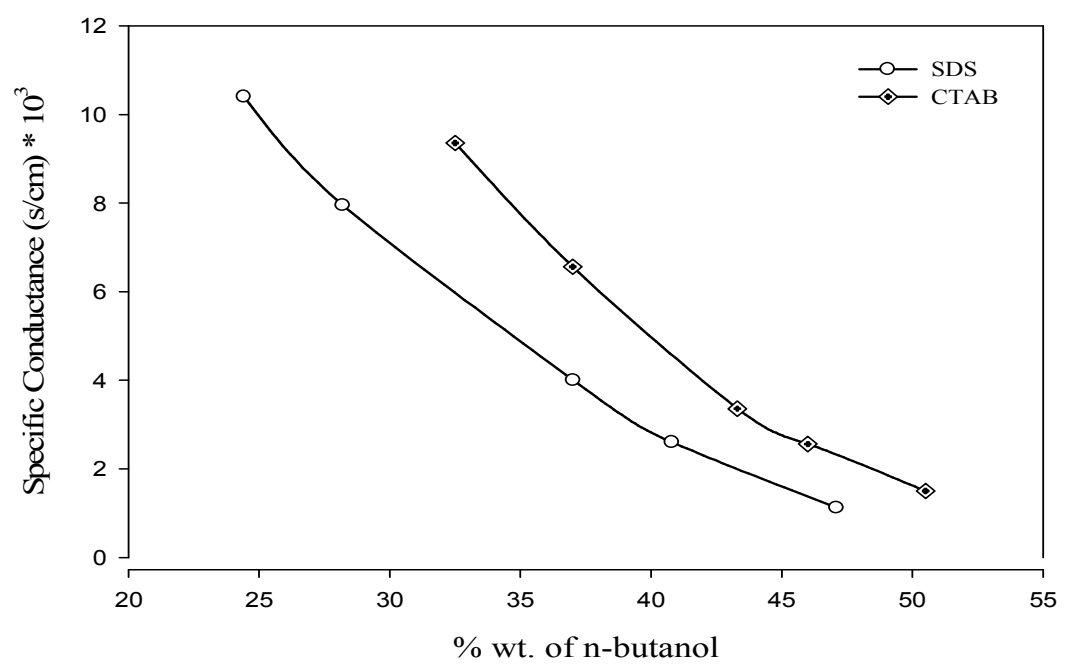

Figure 3. Effect of 1-butanol on the conductivity at varying water to surfactant mole ratio, w0. 


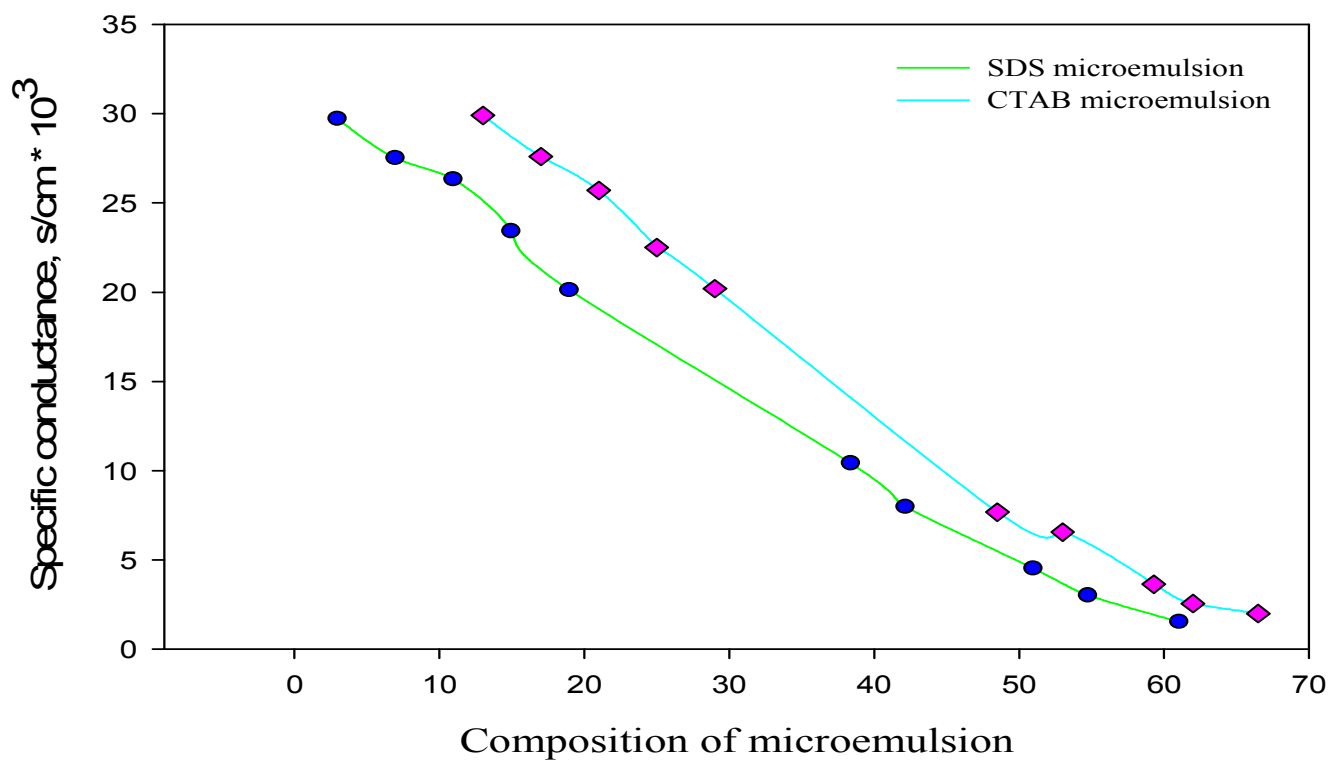

Figure 4. Variation of conductance with composition of SDS and CTAB based microemulsion.
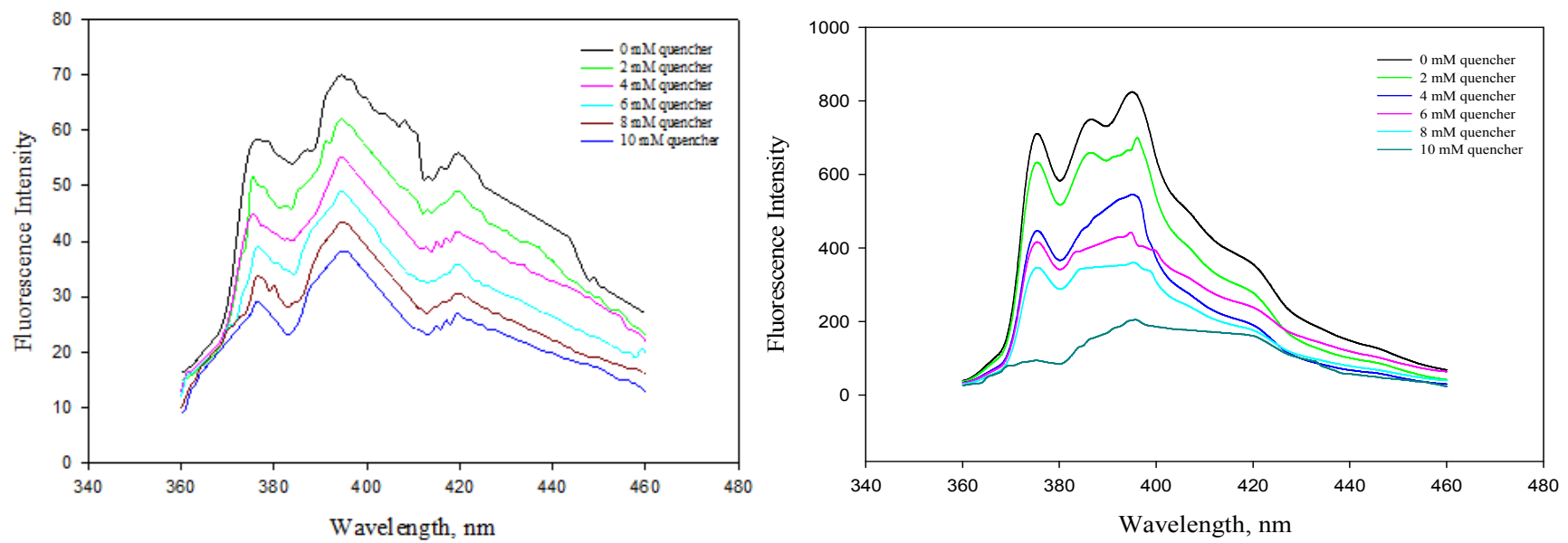

Figure 5. Fluorescence quenching spectra of SDS and CTAB based reverse micelles at $395 \mathrm{~nm}$ by CPC, $N$-cetylpyridiniumchloride.

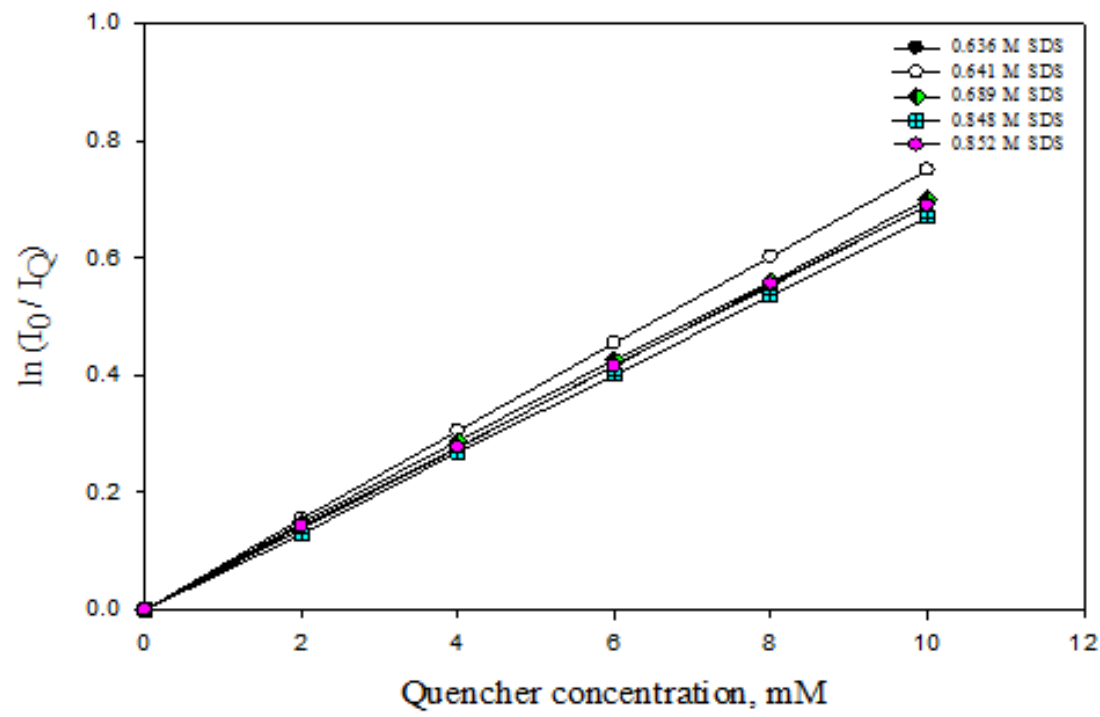




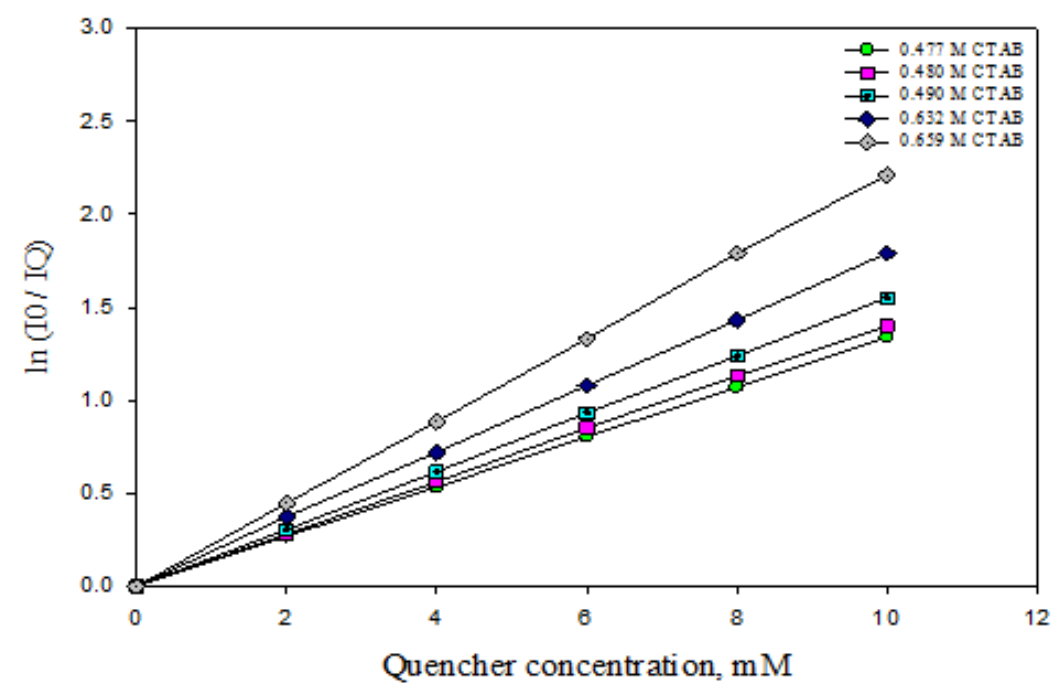

Figure 6. In (IO /IQ) at $395 \mathrm{~nm}$ as a function of concentration of quencher, CPC for different SDS and CTAB concentrations.
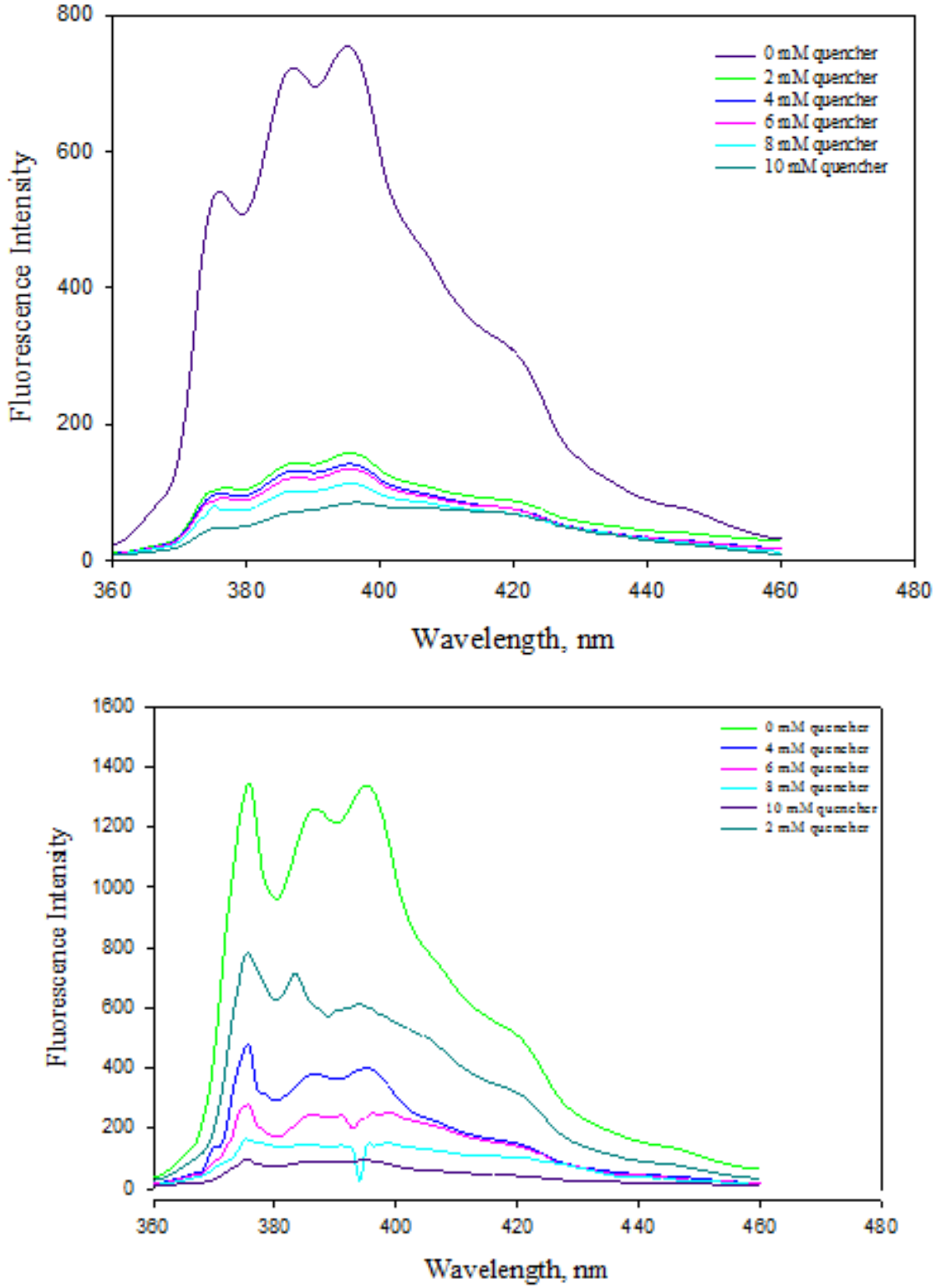

Figure 7. Fluorescence quenching spectra of SDS and CTAB based micelles at $375 \mathrm{~nm}$ by CPC. 

Self-Assembled Aggregates in W/O and O/W Microemulsions as Systems for Preparing Nanomaterials
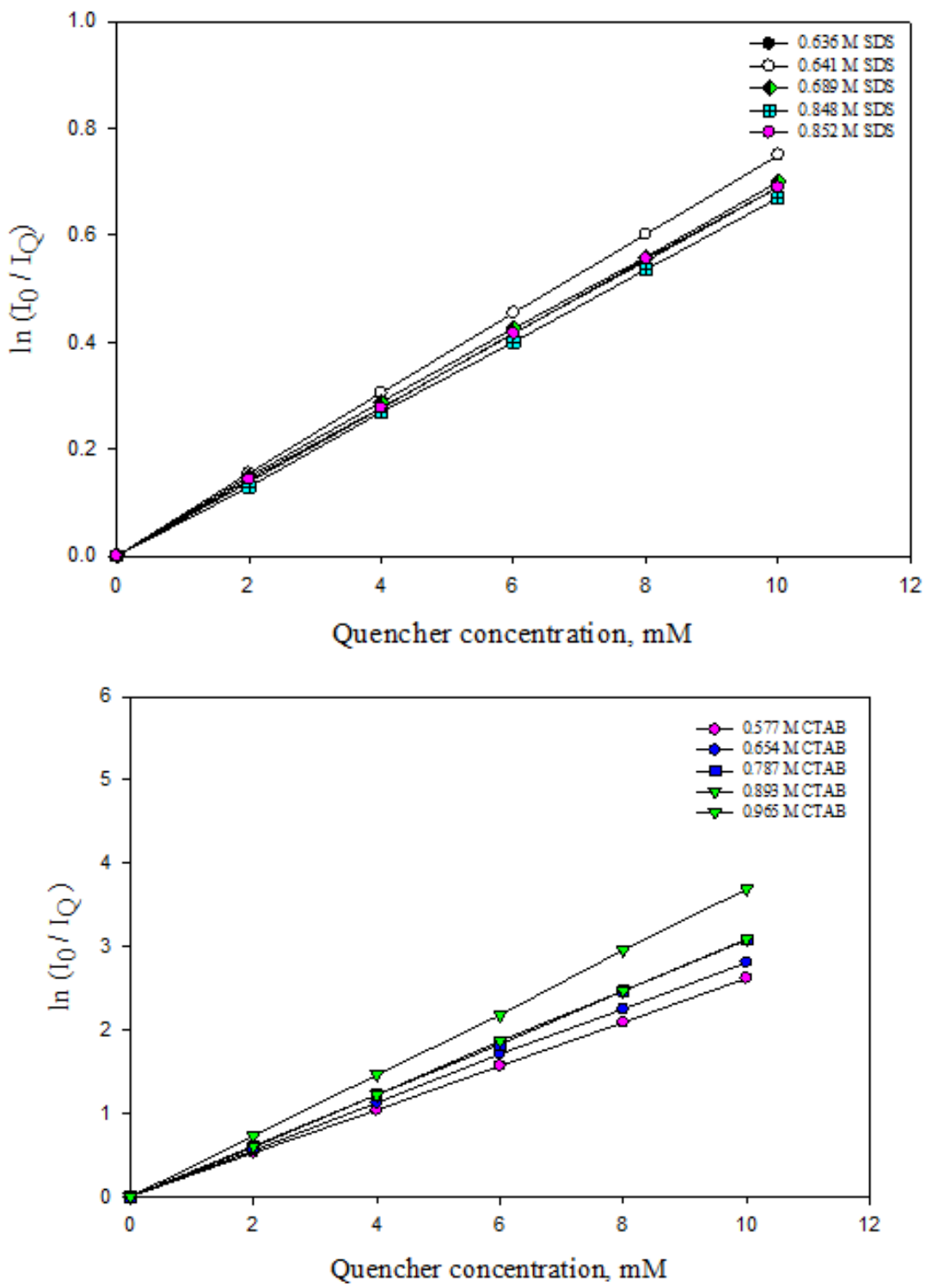

Figure 8. In (I0/IQ) at $375 \mathrm{~nm}$ as a function of concentration of quencher, CPC for different SDS and CTAB concentrations.

Table 12. Reverse cmc of SDS based microemulsion.

\begin{tabular}{lll}
\hline Water to surfactant mole ratio, w0 & Concentration of surfactant present each microemulsion, $\mathbf{M}$ & Reverse cmc, $\mathbf{M}$ \\
\hline 15 & 0.636 & 0.204 \\
20 & 0.641 & 0.213 \\
23 & 0.689 & 0.227 \\
30 & 0.848 & 0.235 \\
33 & 0.852 & 0.293 \\
\hline
\end{tabular}

Table 13. Micellar cme of SDS based microemulsions.

\begin{tabular}{lll}
\hline micelle & Concentration of surfactant present each microemulsion, $\mathbf{M}$ & Micellar cmc, M \\
\hline 1 & 0.754 & 0.4605 \\
2 & 0.823 & 0.595 \\
3 & 0.926 & 0.530 \\
4 & 0.948 & 0.575 \\
5 & 0.964 & 0.607 \\
\hline
\end{tabular}



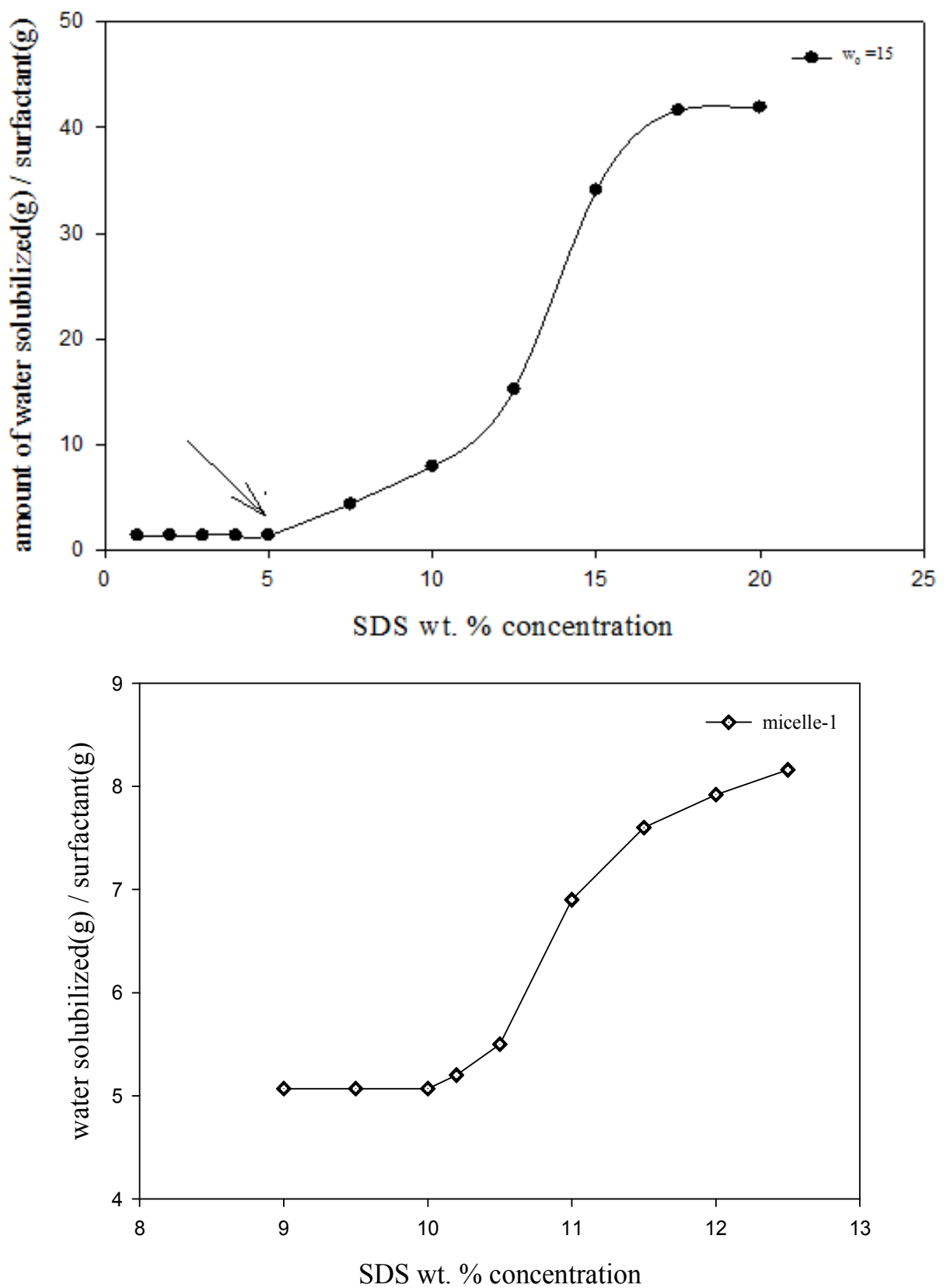

Figure 9. Effect of surfactant concentration on reverse cmc of SDS based w/o microemulsion at w0 15 and cmc of SDS based o/w microemulsion-1.

Table 14. Reverse cmc of CTAB based reverse micelles.

\begin{tabular}{lll}
\hline $\mathbf{w}_{\mathbf{0}}$ & Concentration of surfactant present each microemulsion, $\mathbf{M}$ & reverse cmc, $\mathbf{M}$ \\
\hline 15 & 0.477 & 0.1862 \\
20 & 0.480 & 0.1945 \\
23 & 0.490 & 0.225 \\
30 & 0.632 & 0.337 \\
35 & 0.659 & 0.410 \\
\hline
\end{tabular}

Table 15. Micellarcmc CTAB based micelles.

\begin{tabular}{lll}
\hline micelle & Concentration of surfactant present each microemulsion, $\mathbf{M}$ & Micellar cmc, M \\
\hline 1 & 0.577 & 0.1995 \\
2 & 0.654 & 0.255 \\
3 & 0.787 & 0.345 \\
4 & 0.893 & 0.417 \\
5 & 0.965 & 0.532 \\
\hline
\end{tabular}



Self-Assembled Aggregates in W/O and O/W Microemulsions as Systems for Preparing Nanomaterials
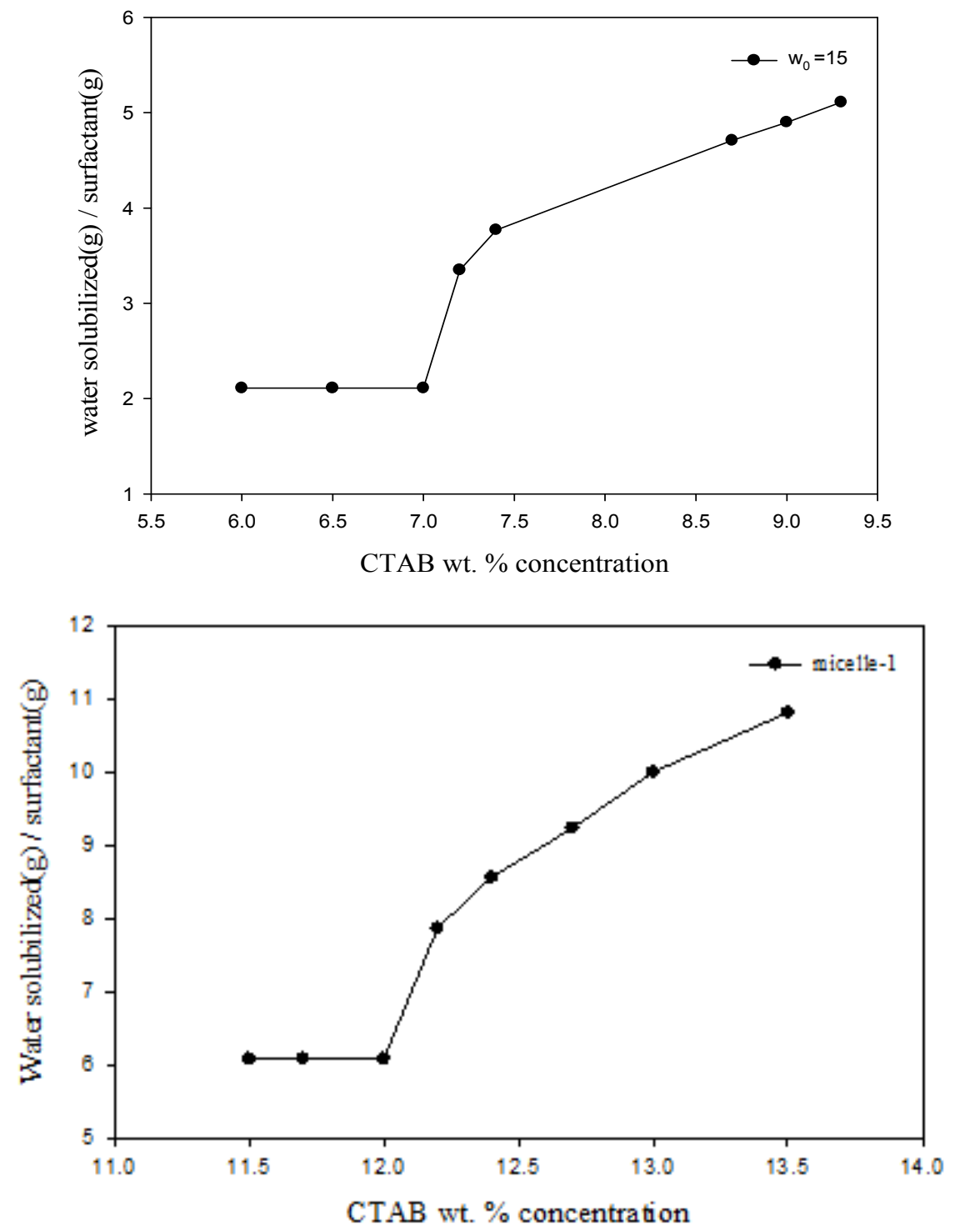

Figure 10. Effect of surfactant concentration on cmc of CTAB based reverse micelle at $w 0=15$.

Table 16. Aggregation numbers of SDS based reverse micelles.

\begin{tabular}{lll}
\hline w0 & Concentration of SDS, $\mathbf{M}$ & Reverse micellar aggregation number, $\mathbf{N}$ \\
\hline 15 & 0.636 & 30 \\
20 & 0.641 & 31 \\
23 & 0.689 & 33 \\
30 & 0.848 & 41 \\
33 & 0.852 & 42 \\
\hline
\end{tabular}

Table 17. Aggregation numbers of SDS based micellar systems.

\begin{tabular}{lll}
\hline Micelles & Concentration of SDS, $\mathbf{M}$ & Micellar aggregation number, $\mathbf{N}$ \\
\hline 1 & 0.754 & 78 \\
2 & 0.823 & 85 \\
3 & 0.926 & 97 \\
4 & 0.948 & 99 \\
5 & 0.964 & 101 \\
\hline
\end{tabular}



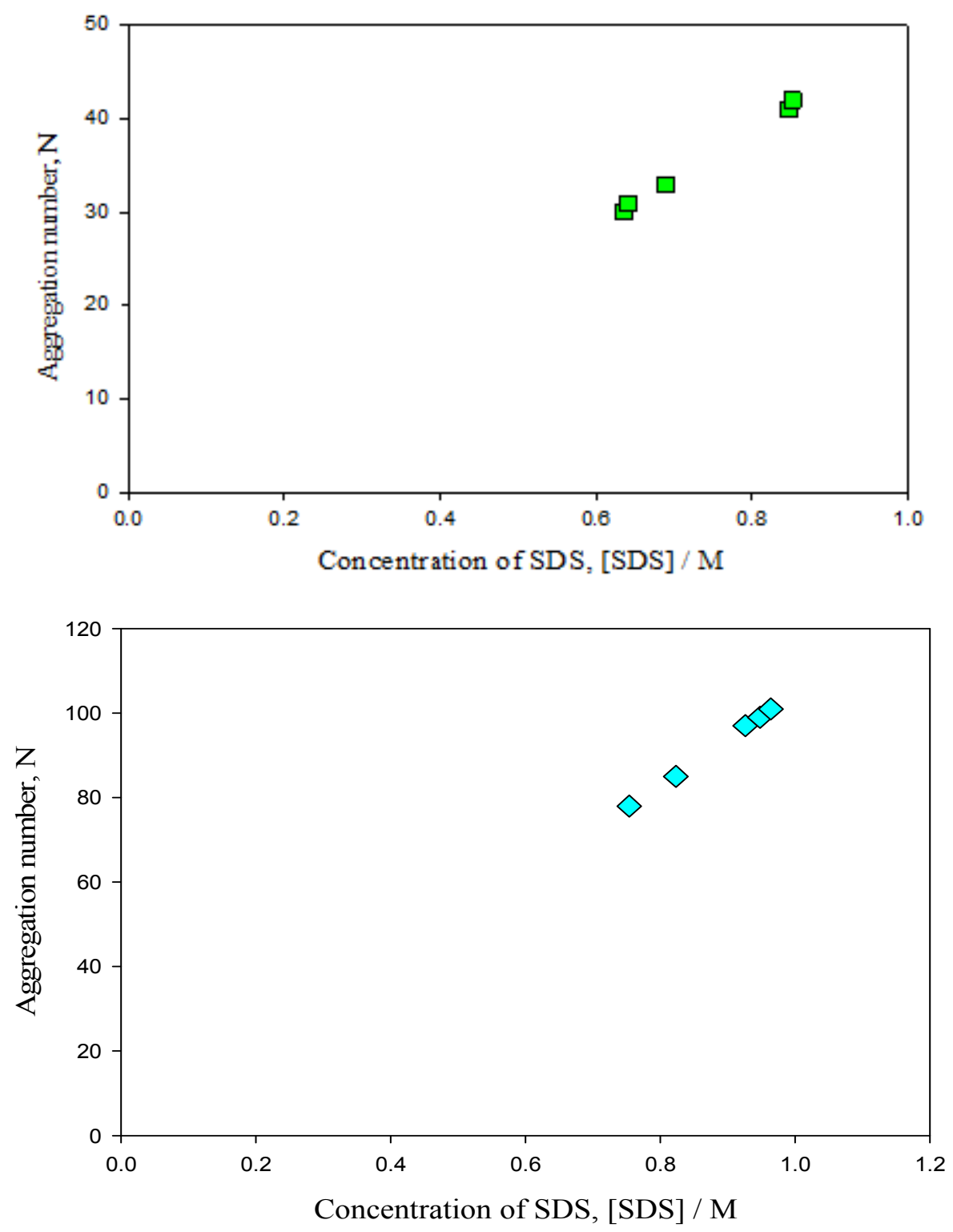

Figure 11. Variation of aggregation number, $N$ with the concentration of SDS based in w/o and o/w microemulsion.

Table 18. Aggregation numbers of CTAB based reverse micelles.

\begin{tabular}{lll}
\hline w0 & Concentration of CTAB, $\mathbf{M}$ & Reverse micellar aggregation number, $\mathbf{N}$ \\
\hline 15 & 0.477 & 39 \\
20 & 0.480 & 40 \\
23 & 0.490 & 41 \\
30 & 0.632 & 53 \\
35 & 0.659 & 55 \\
\hline
\end{tabular}

Table 19. Aggregation number of CTAB based micellar systems.

\begin{tabular}{lll}
\hline micelles & Concentration of CTAB, $\mathbf{M}$ & Micellar aggregation number, $\mathbf{N}$ \\
\hline 1 & 0.577 & 99 \\
2 & 0.654 & 112 \\
3 & 0.787 & 136 \\
4 & 0.893 & 147 \\
5 & 0.963 & 160 \\
\hline
\end{tabular}



Self-Assembled Aggregates in W/O and O/W Microemulsions as Systems for Preparing Nanomaterials
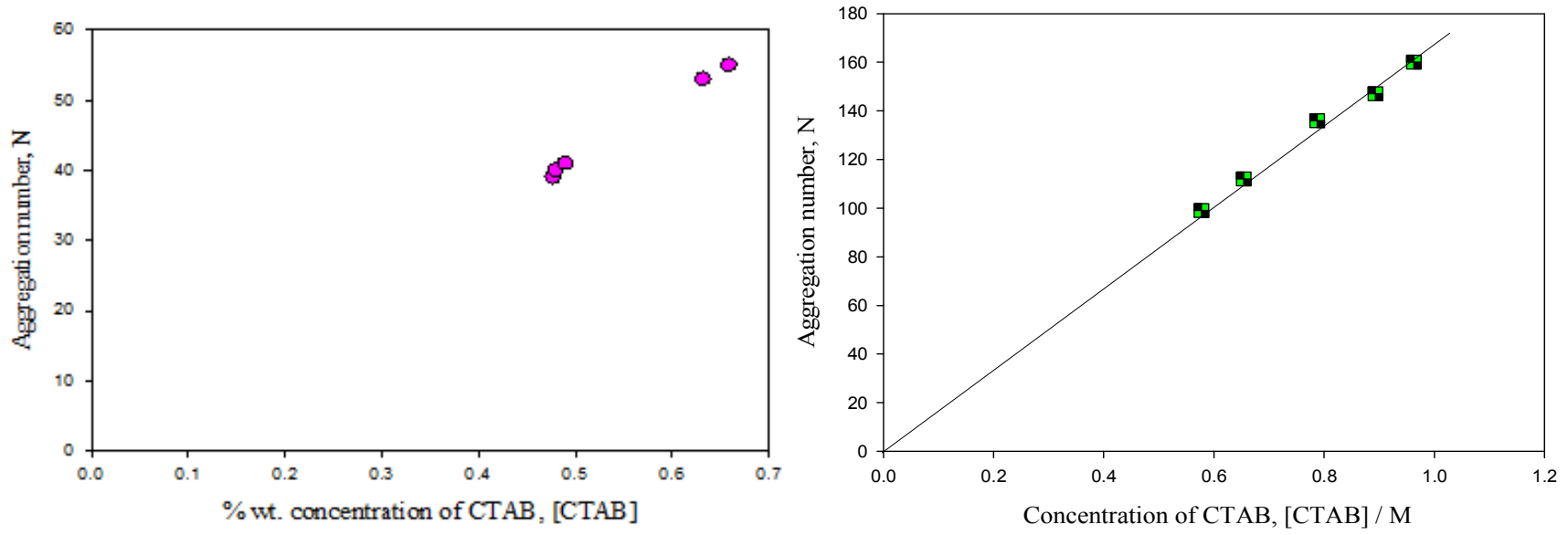

Figure 12. Variation of $N$ with the concentration of CTAB based w/o and o/w microemulsion.
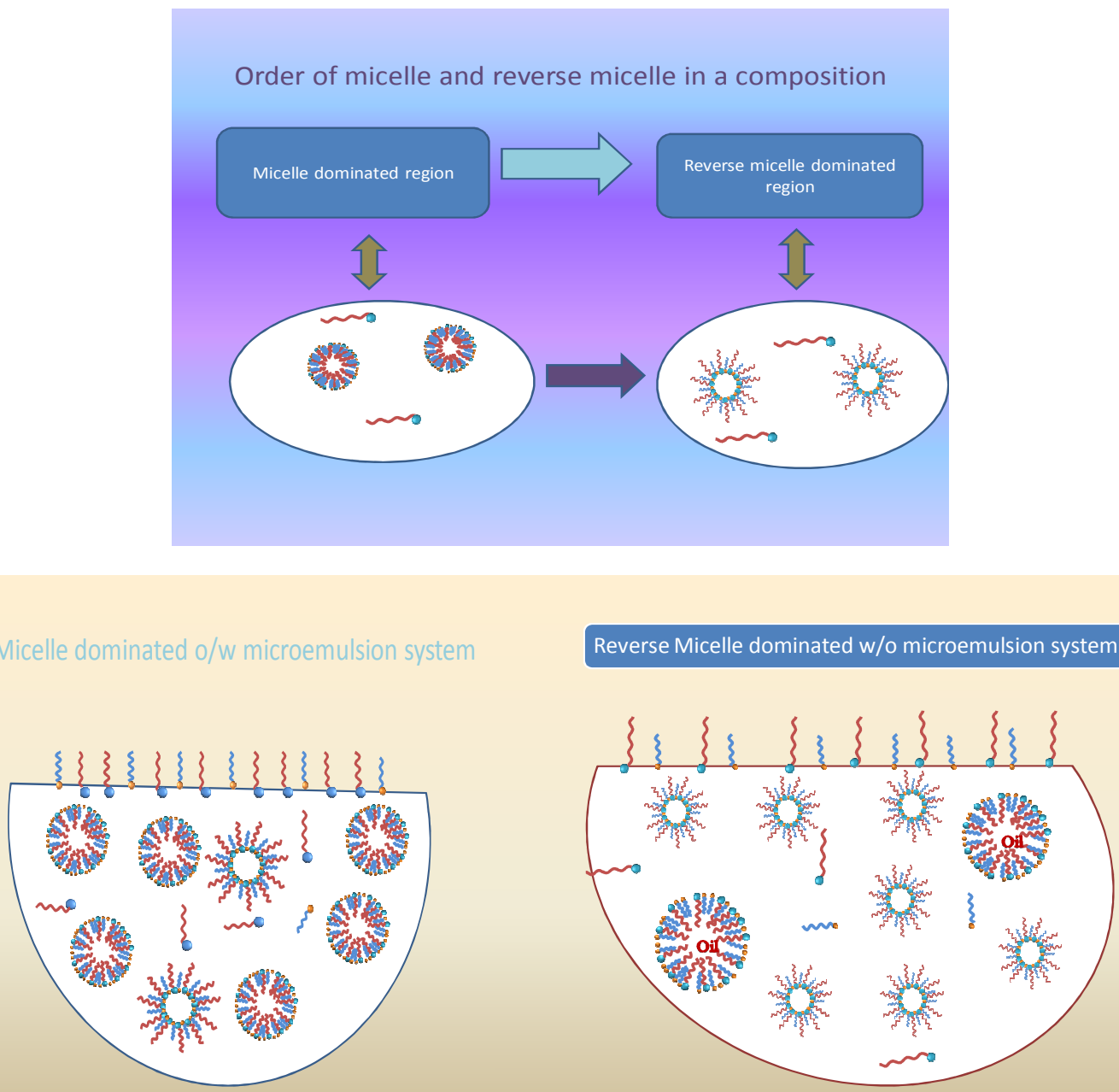

Figure 13. Order of $o / w$ and $w / o$ region and the $w / o$ and $o / w$ microemulsions in a composition of microemulsion. 

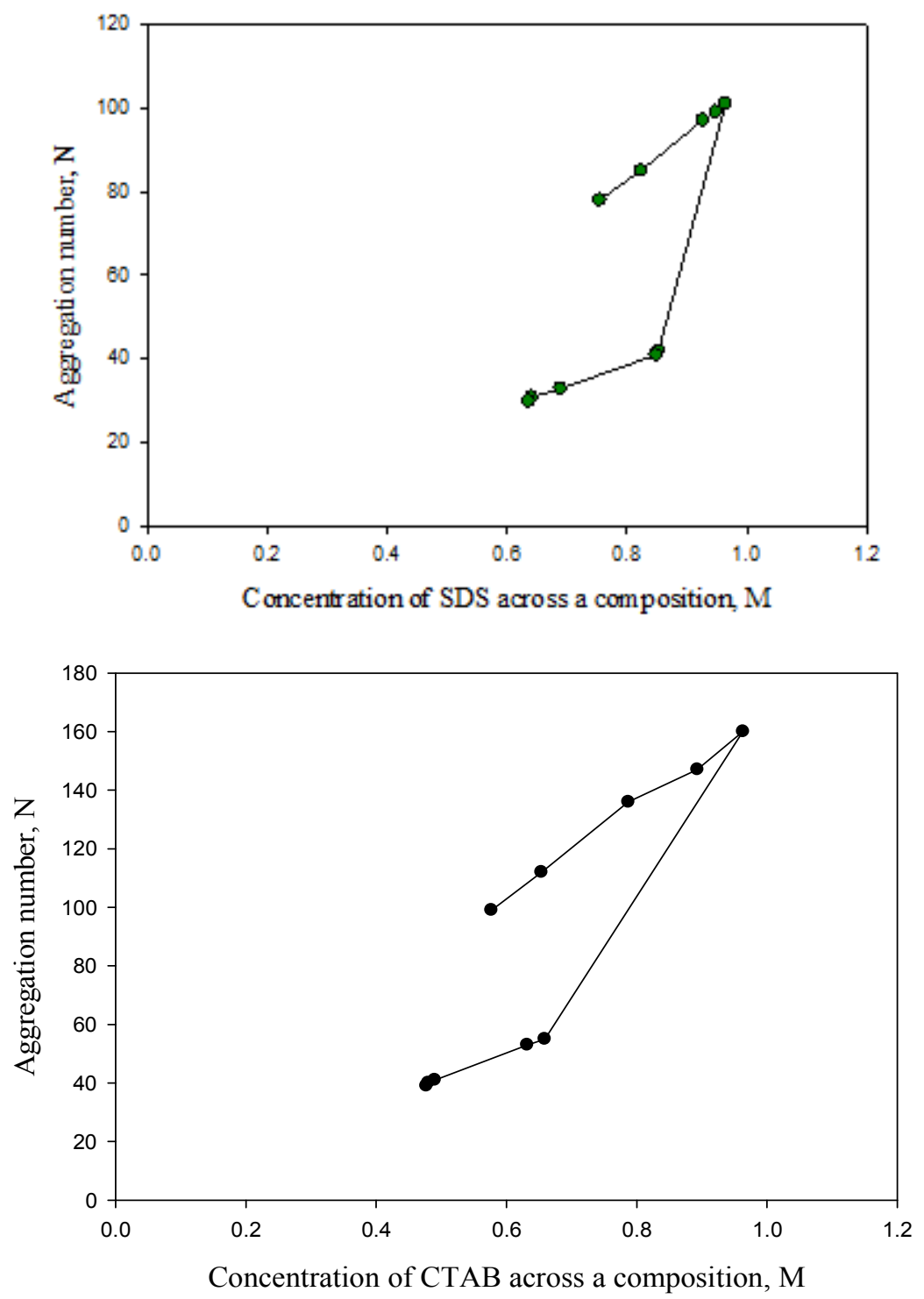

Figure 14. Variation of aggregation number, $N$ with [SDS] and [CTAB] across a composition.

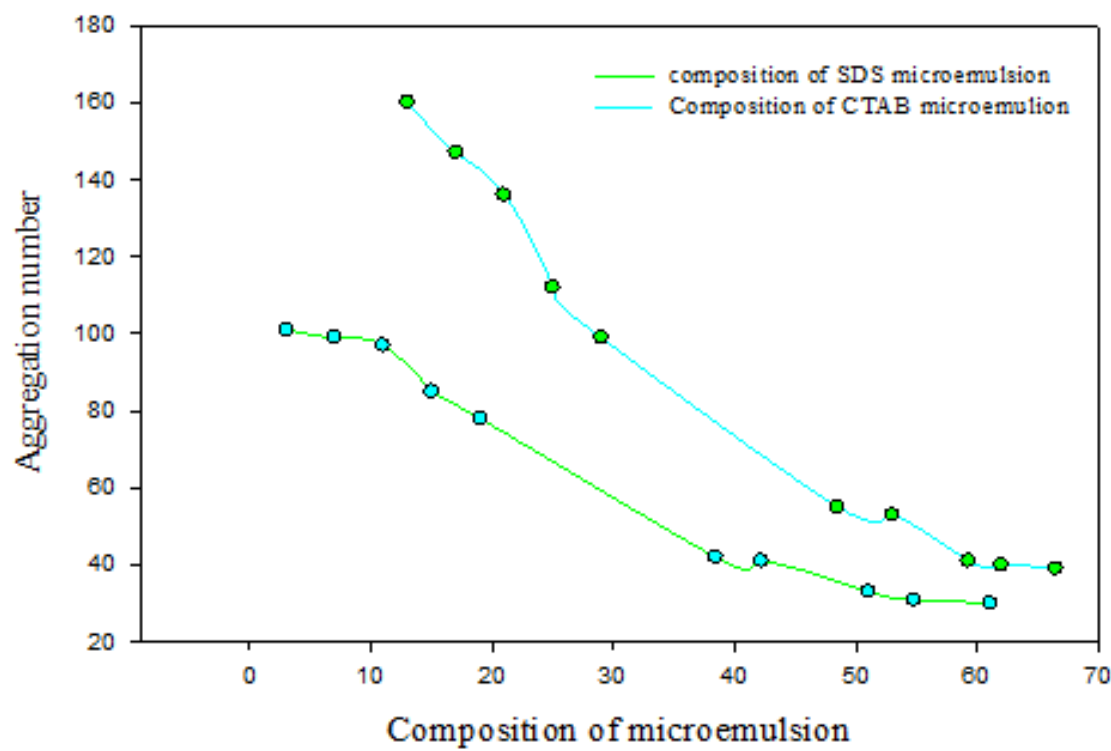

Figure 15. Effect of composition on the aggregation number of SDS and CTAB based microemulsions. 


\section{Conclusions}

As microemulsion technology in worldwide is of major concern, various composition of w/o microemulsion systems with SDS, 1-butanol, cyclohexane, water; CTAB, 1-butanol, cyclohexane, water and tween 80,1-butanol, cyclohexane, water has been prepared at different water to surfactant ratio, w0. And various composition of $\mathrm{o} / \mathrm{w}$ microemulsion systems with SDS, 1-butanol, cyclohexane, water and CTAB, 1-butanol, cyclohexane, water have also been prepared by keeping similarity with the composition of the w/o microemulsion. The composition of microemulsion prepared has been characterized with the help of conductivity, density and aggregation number. And the specific conductance was found to be influenced mainly by the 1-butanol. Higher specific conductances were obtained at lower $\%$ wt.1-butanol while lower specific conductances were obtained at higher $\%$ wt.1-butanol content within a composition. The density was also found to be influenced mainly by the 1-buttanol at similar manner. The aggregation number is dependent on the concentration of surfactant only. As the concentration of the surfactant across the composition is increased, aggregation number increases linearly. However, the corresponding change in aggregation number is not linear to the composition. The aggregation number is increased gradually upto micellar composition, then gradually follows declination upto reverse micellar composition. After the detailed analysis of the conductivity, density and aggregation number, it can be concluded that the size of the micelle and reverse micelle core droplet can be tuned by changing composition of microemulsions. Suitable compositions of microemulsions may be used in detergency, personal care products, cosmetics, micellar catalysis, especially preparation of nanomaterials and so forth.

\section{Acknowledgments}

The author thanks Department of Chemistry, Dhaka University, Bangladesh for the financial support and instrumental support to carry out this work and also thank Dr. Abu Bin Hasan Susan and Dr. Md. Yusuf Ali Mollah, Professors of Dhaka University, Chemistry Department, for supervision, instrumental support and encouragement. The author gratefully acknowledges "Center for Advance Research" for the use of High Resolution Fluorescence Spectrophotometer.

\section{References}

[1] Turner S. R. Siano D. B. and Bock J., "A Microemulsion Process for Producing Acrylamide-Alkyl Acrylamide Copolymers", U. S. Patent No. 4, 1985, vol. 521, pp. 580.

[2] S.P. Moulik, B.K. Paul, Adv. Colloid Interface Sci.(1998),78, 99.
[3] G.B. Behera, B.K. Mishra, P.K. Behera, M. Panda, Adv. Colloid Interface Sci (1998), 82, 1.

[4] J.F. Rusling, Modern Aspects in Electrochemistry, vol. 26, Plenum, New York, 1994, p. 49.

[5] J.F. Rusling, Electroanalytical Chemistry, vol. 18, Dekker, New York, 1994, p. 1.

[6] Promod Kumar, K.L. Mittal (Eds.), Handbook of Microemulsion Science and Technology, Dekker, New York, 1999.

[7] J. Fendler, "Membrane Mimetic Chemistry", Wiley, NY,1982.

[8] Rosen M.J., "Surfactants and Interfacial Phenomena", J. Wiley \& Sons, NY, 1989.

[9] Myers D., "Surfaces, Interfaces and Colloids, Principles and Applications", VCH Pub. Inc., 1991.

[10] Yekta, A., Aikawa, M., Turro N., Chem. Phys. Lett., 1979, 63, 543.

[11] Alexandridis, P.; Athanassiou, V.; Fukuda, S.; Hatton, T. A. Langmuir, 1994, 10, 2604.

[12] Kahlweit, M.; Strey, R.; Busse, G. J. Phys. Chem. 1990, 94, 3881.

[13] Alexandridis, P.; Holzwarth, J. F.; Hatton, T. A. Macromolecules, 1994, 27, 2414.

[14] Alexandridis, P.; Nivaggioli, T.; Hatton, T. A. Langmuir, 1995, $11,1468$.

[15] Alexandridis, P.; Athanassiou, V.; Hatton, T. A. Langmuir, $1995,11,2442$.

[16] Elvira Rodenas' and Mercedes Valiente, "The determination of some physical properties of reverse CTAB micelles in I-hexanol" Colloiris and Surf\&es, (1992), 62, 289-295.

[17] Paschalis Alexandridis*, $\uparrow, \dagger$ and Karin Andersson, "Reverse Micelle Formation and Water Solubilization by Polyoxyalkylene Block Copolymers in Organic Solvent" J. Phys. Chem. B 1997, 101, 8103-8111.

[18] Nivaggioli, T.; Alexandridis, P.; Hatton, T. A.; Yekta, A.; Winnik, M. A. Langmuir, 1995, 11, 730.

[19] Savelli G. ,Germani, R., Brinchi L. , in: J. Texter (Ed.), MarcelDekker, NY, 2001.

[20] Kahlweit, M.; Strey, R.; Busse, G. J. Phys. Chem. 1990, 94, 3881 .

[21] Zana R., "Surfactant Solutions: New Methods for Investigation", Marcell Dekker, NY, 1985.

[22] Alargova R.G., "I.I. ochijashky, M.L. Sierra, R. Za”, Langmuir, 1998, vol. $14,5412$.

[23] Bravo C., Leis J.R., Pe na M.E., J. Phys. Chem., 1992, vol. 96, pp. 1957.

[24] N.J. Turro, A. Yekta, J. Am. Chem. Soc. 100 (1978), 5951.

[25] A. Yekta, M. Aikawa, N. Turro, Chem. Phys. Lett. 63 (1979), 543. 
[26] P.K. Behera, A.K. Mishra, J. Photochem. Photobiol. A: Chem. 71 (1993), 115.

[27] M. Sirish, B.G. Maiya, J. Photochem. Photobiol. A: Chem. 85 (1995), 127.

[28] Kahlweit, M.; Strey, R.; Busse, G. J. Phys. Chem., 1990, 94, 3881 .
[29] E. Rcdenas and E. Perez-Benito. J. Phys. Chem., (1991),95, 4452.

[30] N. Lufimpadio. J.B. Nagy and E.G. De Rouane. in K.I. Mittal and B. Lindman (Eds), Surfactants in Solution,Vol. 3, Plenum Press, New York, 1984, p.1483. 